\title{
Attitudes des médecins généralistes envers les médecines complémentaires et besoins de formation. Résultats d'une enquête suisse
}

\author{
Attitudes of general practitioners toward complementary medicine \\ and training needs assessment. \\ Results of a Swiss survey
}

Selon l'Organisation mondiale de la santé, les médecines complémentaires (MC) « se rapportent à un vaste ensemble de pratiques de soins de santé qui n'appartiennent pas à la tradition du pays et ne sont pas intégrées dans le système de santé dominant ${ }^{[1]}{ }$. Comme dans d'autres pays occidentaux, les MC sont aujourd'hui très utilisées en Suisse. D'après l'enquête suisse sur la santé 2007, $31 \%$ de la population du canton de Vaud y a fait appel au moins une fois au cours de l'année précédant l'analyse ${ }^{[2]}$. Wolf et al. ${ }^{[3]}$ ont mis en évidence une prévalence d'utilisation en Suisse de $49 \pm 22 \%$. Ces médecines sont largement recherchées par les patients en complément à la médecine conventionnelle, bien que $77 \%$ d'entre eux ne disent pas à leur médecin généraliste qu'ils y recourent ${ }^{[4]}$. Par ailleurs, Déglon-Fischer et al. ${ }^{[5]}$ ont montré que la plupart des médecins généralistes sont ouverts à ce sujet et que les $\mathrm{MC}$ jouent un rôle important dans la prise en charge des patients.

Dans de nombreux pays, le besoin d'un enseignement relatif aux $\mathrm{MC}$ a été reconnu ${ }^{[6]}$. Actuellement en Suisse, les universités ne proposent pas de formation en MC mais la plupart des facultés de médecine ont commencé à sensibiliser les étudiants aux phénomènes d'utilisation des $\mathrm{MC}$ et de noncommunication de leur usage ${ }^{[7]}$.
Peu d'études se sont intéressées aux connaissances que les médecins généralistes avaient des MC. En Californie, $61 \%$ des médecins estiment n'avoir pas assez de connaissances sur la sécurité ou l'efficacité des MC et $81 \%$ aimeraient recevoir davantage de formation $^{[8]}$. La Mayo Clinic aux États-Unis s'est intéressée en 2006 à l'attitude de ses internistes faces aux $\mathrm{MC}$ et à leurs connaissances sur ce sujet ${ }^{[9]}$ : la plupart a admis ne pas être à l'aise pour conseiller des patients sur les MC et éprouver de grandes difficultés à trouver des informations fiables sur leur utilisation.

Nous avons conduit une enquête auprès des médecins généralistes installés dans le canton de Vaud (Suisse), visant à décrire les attitudes et les connaissances des généralistes vaudois à l'égard des $\mathrm{MC}$ et à identifier leurs besoins perçus de formation dans ce domaine.

Dans un premier temps, un questionnaire a été administré en mars 2012 lors d'une matinée de formation continue - les « Jeudis de la Vaudoise »organisée par la Société vaudoise de médecine, sur le thème des MC. Parmi les répondants (109/182 soit $59,9 \% ; 58,3 \%$ d'hommes), 29,6\% avaient eu leur diplôme avant 1980, 28,7\% entre 1980 et 1989, $19,4 \%$ entre 1990 et 1999 , et $22,2 \%$ après 2000 ; $69,2 \%$ n'avaient pas de formation en MC, sans 
différence significative selon le genre ou l'expérience. Des entretiens semi-directifs ont ensuite été menés entre juillet et octobre 2012 auprès de dix médecins généralistes vaudois, sollicités sur la base de leur volontariat exprimé lors de la matinée de formation continue ou recrutés au sein du réseau professionnel des enquêteurs (sept hommes et trois femmes, tous diplômés avant 1986). Le guide d'entretien avait été élaboré à partir des réponses au premier questionnaire. Enfin, un deuxième questionnaire, administré anonymement sur internet en octobre 2012, à partir d'un lien envoyé par courrier électronique aux 600 généralistes vaudois inscrits auprès de l'association vaudoise des médecins de famille (MF-Vaud), n'a recueilli qu' un faible taux de réponse ( $45 / 600$ soit 7,5\%; 71,1\% d'hommes). Les questionnaires et la grille d'entretiens ont été adaptés au contexte local à partir d'études précédentes ${ }^{[7,9]}$. Les questionnaires ont été lus de manière automatique. Les entretiens ont été analysés par catégorisation thématique (catégories prédéterminées et catégories émergentes) des réponses, au fur et à mesure des entretiens, ce qui a permis de vérifier la saturation des données.

Au décours immédiat de la séance de formation continue consacrée aux $\mathrm{MC}, 17 \%$ des répondants disaient savoir où trouver une information factuelle et $75 \%$ n'avaient à cet égard que des informations parcellaires. Par ailleurs, les ressources exploitées pour conseiller les patients restent assez limitées : les interrogés évoquent des lectures ou des discussions entre collègues, mais ne recourent pas à des bases de données formelles. Pourtant, plusieurs ressources ayant fait l'objet d'une validation académique et mises à jour régulièrement sont disponibles ${ }^{[11-13]}$. Aucun médecin reçu en entretien n'avait reçu une information de source académique durant son cursus ; toutes les connaissances en MC avaient été acquises selon l'intérêt personnel, en général en fonction d'un cas concret. Les besoins et attentes de formation étaient clairement formulés : $53,6 \%$ des répondants désiraient en savoir plus sur la recherche, $50,9 \%$ sur les pratiques complémentaires (ostéopathie, homéopathie, etc.), 70,9\% sur l'application des MC à certaines pathologies (dépression, fibromyalgie, etc.). Seuls 3,6\% ne désiraient aucune nouvelle formation.
Concernant la formation initiale, les entretiens révélaient une préférence pour une sensibilisation en formation pré-graduée plutôt qu'une formation très spécifique sur certaines MC ; les participants suggéraient notamment d'encourager les médecins à rechercher systématiquement le recours à des $\mathrm{MC}$ au cours de l'anamnèse. Quatre-vingt dix pour cent des répondants au questionnaire par internet pensaient qu'il faut informer les médecins, soit par des cours de sensibilisation (fréquence d'utilisation, interactions, ...), soit par des cours fournissant des informations de base (définition de quelques MC, indications, limites, ...).

En formation continue, les interrogés privilégiaient des approches contextualisées des MC lors de la discussion sur la prise en charge d'un patient souffrant d'une certaine pathologie spécifique (exemple : pour les douleurs chroniques, aborder le traitement standard, mais aussi l'hypnose ou l'acupuncture).

Une très forte majorité des répondants au premier questionnaire $(91,8 \%)$ pensait que la mise à disposition d'une consultation d'orientation et de conseil sur les MC dans le canton pourrait aider leurs patients. Ces médecins estimaient que $25 \%$ de leurs patients étaient concernés.

Les médecins reçus en entretiens abordaient peu la question du recours à des MC avec leurs patients ; deux sur dix y sont très opposés mais la même proportion proposent à leurs patients d'y recourir, notamment lorsqu'ils n'arrivent pas à soulager suffisamment le patient avec la médecine conventionnelle.

En dépit de ses limites (taux de réponse au deuxième questionnaire très faible, biais de sélection pour chacune des trois approches), notre enquête met en évidence un intérêt très significatif des médecins répondants à l'égard des MC. L'enseignement dispensé actuellement à l'Université de Lausanne est essentiellement fondé sur les résultats de la recherche épidémiologique et clinique. Cette orientation semble correspondre aux intérêts des médecins répondants de cette enquête (la moitié demandaient davantage d'informations relatives à la recherche dans le domaine des MC) ce qui rejoint les conclusions de plusieurs enquêtes et groupes de travail ${ }^{[8,14,15]}$. Des données scientifiques de qualité sont aujourd'hui 
disponibles ${ }^{[11-13]}$. Plusieurs études ou rapports proposent qu' un tel enseignement soit obligatoire en formation pré-gradué ${ }^{[5,16]}$, sous forme de cours ou de séminaire ${ }^{[7]}$, et qu'il porte notamment sur la nécessité d'aborder systématiquement les MC durant l'anamnèse ${ }^{[9,17]}$. Compte tenu de la forte prévalence de recours à la $\mathrm{MC}$ par les patients et des données scientifiques, la place des MC devrait être également plus abordée lors de la formation post-graduée ${ }^{[5,16]}$.

À l'Université de Lausanne comme dans d'autres centres universitaires, des cours de sensibilisation sur les MC commencent à être proposés en formation pré-graduée et post-graduée. Certaines prestations de MC sont proposées à l'hôpital, par exemple l'hypnose et l'acupuncture pour la douleur. L'évaluation de ces innovations reste toutefois encore souvent insuffisante, au regard de la nécessité de garantir la neutralité, la rigueur scientifique et la nécessité de mise à jour ${ }^{[18]}$.

En conclusion, cette enquête confirme un intérêt important des médecins généralistes pour qu'un enseignement sur les MC soit introduit dans la formation pré-graduée dans les facultés de médecine, mais également dans la formation post-graduée et continue, afin d'aider le patient à effectuer des choix correctement informés. La capacité des médecins généralistes à informer leurs patients sur la base de connaissances factuelles dans le domaine des MC permettrait de fait d'optimiser la prise en charge et d'éviter certaines dérives. Il importe en effet que les médecins puissent proposer tout traitement ayant fait ses preuves, potentiellement bénéfique pour le patient, et à l'inverse, qu'ils soient en mesure de formuler des réserves argumentées à l'égard d'approches potentiellement délétères.

\section{Sabina SCHNEIDER Bertrand GRAZ Pierre-Yves RODONDI Eric BONVIN}

Unité de recherche et d'enseignement sur les médecines complémentaires Centre hospitalier universitaire vaudois - Université de Lausanne, Suisse Mailto : Sabina.Schneider@unil.ch

\section{Références}

1. Organisation mondiale de la santé. Médecine traditionelle: définitions. WHO. [On-line] Disponible sur: http://www.who.int/topics/ traditional_medicine/definitions/fr/index.html

2. Observatoire suisse de la santé (Obsan). La santé dans le canton de Vaud, Enquête suisse sur la santé 2007. [On-line] Disponible sur: http://www.bfs. admin.ch/bfs/portal/fr/index/news/publikationen.Document.138757.pdf

3. Wolf U, Maxion-Bergemann S, Bornhöft G, Matthiessen PF, Wolf M. Use of complementary medicine in Switzerland. Forsch Komplementmed. 2006;13 (Suppl 2):4-6.

4. Robinson A, McGrail MR. Disclosure of CAM use to medical practitioners: a review of qualitative and quantitative studies. Complement Ther Med 2004; 12:90-8.

5. Déglon-Fischer A, Barth J, Ausfeld-Hafter B. Komplementärmedizin in Schweizer Praxen der Grundversorgung. Forsch Komplementmed 2009; 16:251-5.

6. Frenkel M, Ben Arye E. The growing need to teach about complementary and alternative medicine: questions and challenges. Acad Med 2001;76:251-4.

7. Nicolao M, Täuber MG, Heusser P. How should complementary and alternative medicine be taught to medical students in Switzerland? A survey of medical experts and students. Med Teach 2010;32:50-5.

8. Milden SP, Stokols D. Physicians' attitudes and practices regarding complementary and alternative medicine. Behav Med 2004;30:73-82.

9. Wahner-Roedler DL, Vincent A, Elkin PL, Loehrer LL, Cha SS, Bauer BA. Physicians' attitudes toward complementary and alternative medicine and their knowledge of specific therapies: a survey at an academic medical center. Evid Based Complement Alternat Med 2006;3:495-501.

10. Existe-t-il des données scientifiques sur l'efficacité clinique des médecines complémentaires?[On-line] Disponible sur: http://www.medicalforum.ch/ pdf/pdf_f/2011/2011-45/2011-45-242.PDF

11. CAM on PubMed ${ }^{\circledR}$ [On-line]. Disponible sur: http://nccam.nih.gov/research/camonpubmed 
12. The Cochrane Collaboration. Trusted evidence. Informed decisions. Better health. [On-line]. Disponible sur: http://www.cochrane.org/

13. Marcus DM. How should alternative medicine be taught to medical students and physicians? Acad Med 2001;76:224-9.

14. Kligler B, Maizes V, Schachter S, Park CM, Gaudet T, Benn R, et al. Core competencies in integrative medicine for medical school curricula: a proposal. Acad Med 2004;79:521-31.
15. Varga O, Márton S, Molnár P. Status of complementary and alternative medicine in European medical schools. Forsch Komplementmed 2006;13:41-5.

16. Featherstone C, Godden D, Selvaraj S, Emslie M, Took-Zozaya M. Characteristics associated with reported CAM use in patients attending six GP practices in the Tayside and Grampian regions of Scotland: a survey. Complement Ther Med 2003;11:168-76.

17. Marcus DM, McCullough L. An evaluation of the evidence in "evidence-based" integrative medicine programs. Acad Med 2009;84:1229-34. 